\title{
FPGA-based high resolution DPWM control circuit
}

\author{
SONG Hu${ }^{1,2}$, JIANG Naiti², HU Shanshan ${ }^{1}$, and LI Hongtao ${ }^{1, *}$ \\ 1. School of Electronic and Optical Engineering, Nanjing University of Science and Technology, Nanjing 210094, China; \\ 2. Nanjing Marine Radar Institute, Nanjing 210003, China
}

\begin{abstract}
Two improved structures of high resolution digital pulse width modulator (DPWM) control circuit are proposed. Embedded digital clock manager (DCM) blocks and digital programmable delay circuits are employed as the basic resources to construct the field-programmable gate array (FPGA)-based DPWM implementations. Detailed schemes are illustrated and the circuits have been successfully implemented on the Artix-7 FPGA device developed by Xilinx. Experimental results show that when the basic clock operates at the frequency of $200 \mathrm{MHz}$, the resolutions of the two approaches can reach 625 ps and 500 ps, respectively. Besides, the presented schemes possess other merits including flexible resolution, strong versatility and relatively good stability.
\end{abstract}

Keywords: digital clock manager (DCM), digital programmable delay circuit, digital pulse width modulator (DPWM).

DOI: $10.21629 / J S E E .2018 .06 .03$

\section{Introduction}

In recent years, digital pulse width modulator (DPWM) has been widely used in the fields of electronic engineering, such as power converters, digital radar systems [1 -7], etc. For instance, the performance of the power converter is closely related to the DPWM since its resolution directly affects the accuracy of the output voltage and current [2]. Besides, high resolution DPWM also provides a feasible approach for broadband digital beamforming in array radar systems.

Compared with traditional analog solutions, DPWM control circuit has many significant advantages, such as strong configurability, flexible applications of advanced control algorithms and so on [8-12]. In order to obtain mature high resolution DPWM, experts from different research institutions have presented various design schemes in existing studies, including counter-based, digital

Manuscript received November 06, 2017

*Corresponding author.

This work was supported by the National Natural Science Foundation of China (61401204), the Fundamental Research Funds for the Central Universities (30916011319), the Technology Research and Development Program of Jiangsu Province (BY2015004-03), and the Postdoctoral Science Foundation of Jiangsu Province (1501104C). clock manager (DCM) based and IODELAY-based approaches [13-18]. The common ground of these solutions is that field-programmable gate array (FPGA) devices are employed for engineering implementations. For instance, a DCM-based DPWM scheme was presented and analyzed in [14]. However, the applicability of the method is poor because of the instability and the limited resolution. It is reported that IODELAY-based DPWM can reach the resolution of 78 ps [15]. However, one thing to note is that IODELAY elements only exist in high-end FPGA series up to now, which in turn implies that the scheme has the disadvantage of high cost.

This paper focuses on DPWM implementations realized by commercial digital control circuits. In particular, two FPGA-based structures for high-resolution DPWM are proposed, where the first approach is also based on DCM, but the RS latch utilized in previous work is replaced by the OR gate which possesses good stability as well as simplicity. As for the second method, look-up-table (LUT) is employed as the basic resources to construct high resolution DPWM. The most appealing aspect of the scheme is that the significantly simplified structure can be easily implemented on low-cost FPGA devices.

This paper is organized as follows. Section 2 and Section 3 elaborate on the structures of the proposed DPWM control circuits. In Section 4, detailed implementation and experimental results based on the Artix-7 FPGA devices are presented and analyzed. Finally, Section 5 concludes the paper.

\section{DPWM based on embedded DCM}

\subsection{Overview}

The starting point of the first method is to precisely control the delay of the pulse signals by using the rising and falling edges of clocks with fixed phase differences. Then, the original and the delayed pulse signals are input into the OR gate to get the desired DPWM signal. Obviously, the rising edge of the original pulse determines the rising edge 
of the DPWM signal, and the falling edge of the delayed signal determines the falling edge of the DPWM signal. To be more specific, the proposed DPWM consists of clock generation circuit, pulse width generation circuit, phaseshift delay circuit and gate circuit, where the clock generation circuit is implemented by the embedded DCM modules. Typically, the structure of the embedded DCM modules in FPGA devices have the following functions [19]:

(i) Phase shift: DCM usually can provide four phaseshift clock signals compared to the reference clock signal CLKIN, i.e., CLK0, CLK90, CLK180, and CLK270 with phase shift of $0^{\circ}, 90^{\circ}, 180^{\circ}$ and $270^{\circ}$, respectively. In DCM configuration, the relative phase shift can also be set to be variable. For instance, if the range of the PHASE_SHIFT attribute is [-255, 255], then a phase shift of PHASE_SHIFT $\times T / 256$ can be achieved with respect to CLKIN, where $T$ is the clock cycle.

(ii) Frequency synthesis: frequency multiplication and division of DCM can produce a wide range of output frequencies which will be denoted as CLKFX hereafter.

Besides, feedback clock signal CLKFB is used to compare the output signal and the input signal. When the lock loop enters into the "lock" state, the input clock and CLKFB will keep synchronization, as long as the input clock does not change.

\subsection{Generalization of the DCM-based architecture}

The main subject of this subsection is to demonstrate the scheme of the DPWM shown in Fig. 1. As mentioned earlier, the generalized DPWM structure consists of four blocks, the operational process of which can be depicted as follows.

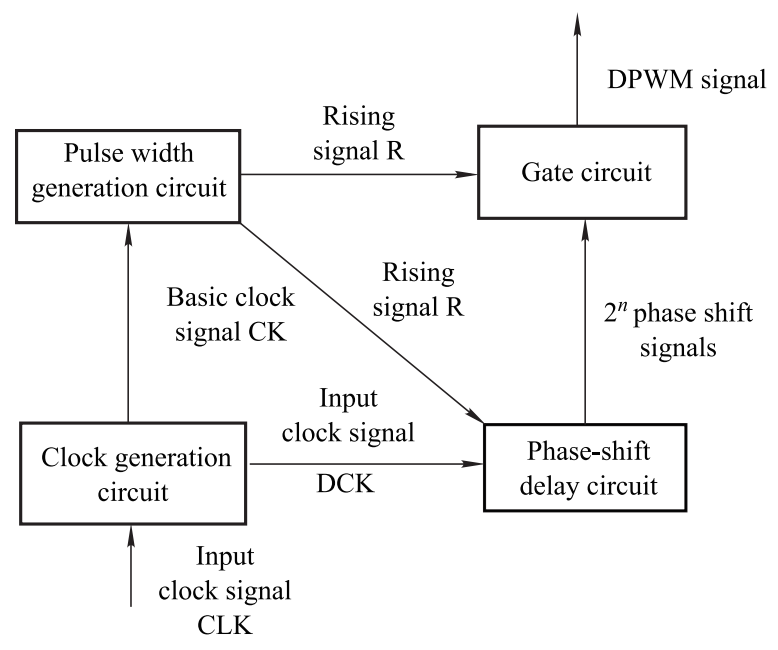

Fig. 1 Workflow of the DCM-based DPWM

(i) Clock generation circuit generates the basic clock signal CK and the delayed clock signal DCK, where CK and DCK respectively serve as the clock signal of the pulse width generation circuit and the phase-shift delay circuit.

(ii) Pulse width generation circuit produces the rising signal R which will be used as the input signal of the phaseshift delay circuit and the gate circuit.

(iii) The phase-shift delay circuit shifts the input signal $\mathrm{R}$ and outputs eight phase-shifting signals.

(iv) The selector in the gate circuit chooses one of the eight phase-shifting signals as the falling signal $\mathrm{F}$.

(v) Connect the rising and falling signals to the input ports of the OR gate and output the desired DPWM signal (see Fig. 2).

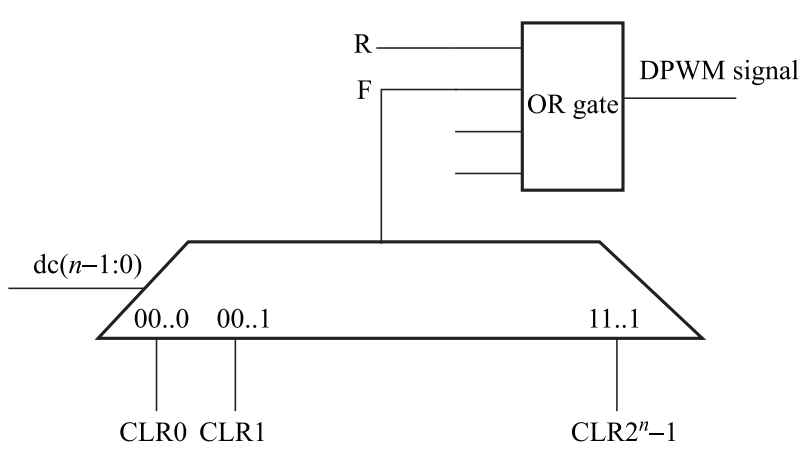

Fig. 2 The structure of the gate circuit

Notice that the proposed DPWM works under the control of the duty cycle command (DCC) signal $\mathrm{dc}(m: 0)$, where $\operatorname{dc}(m: n)$ is the most significant bits (MSBs) and $\mathrm{dc}(n-1: 0)$ is the least significant bits (LSBs). The MSBs serve as the control word of the pulse width generation circuit, and the LSBs are used as the control word of the selector in the gate circuit. Essentially, MSBs decide the pulse width of the rising signal R and LSBs control its decimal delay with respect to the basic clock cycle. Theoretically, the frequency of the DPWM signal is $1 /\left(2^{m-n+1} T\right)$ and the resolution is $T / 2^{n}$, where $T$ is the period corresponding to the basic clock signal CK. As indicated in Fig. 3, the rising edge of signal $\mathrm{R}$ determines the rising edge of the DPWM signal, and the falling edge of signal $F$ determines the falling edge of the DPWM signal.

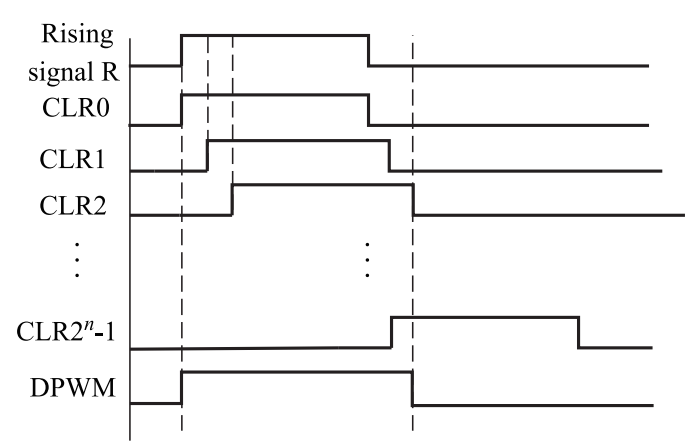

Fig. 3 Timing flow of the gate circuit 
In the sequel, we elaborate more on the implementation of the DPWM, where the case that $[m, n]=[10,3]$ is taken as an example. As is shown in Fig. 4(a), the clock generation circuit involves three embedded DCMs, where DCM2 is utilized to produce the desired basic clock signal. A typical configuration is that the input clock signal CLK is set to $50 \mathrm{MHz}$ and the basic clock signal CK is $200 \mathrm{MHz}$ after quadrupling operation. DCM0 and DCM1 produce a set of delayed signals by phase shifting CK, namely DCK $=\{\mathrm{CK} 0, \mathrm{CK} 1, \mathrm{CK} 2, \mathrm{CK} 3\}$. To be specific, the PHASE-SHIFT attribute of DCM0 is set to 0 and it outputs the delayed clock signal CK0 and CK2 with phase shift of $0^{\circ}$ and $90^{\circ}$. Similarly, the PHASE-SHIFT attribute of DCM1 is set to 32 and it outputs the delayed clock signal CK1 and CK3 with phase shift of $45^{\circ}$ and $135^{\circ}$.

We next illustrate the structure of the pulse width generation circuit appearing in Fig. 1. The pulse width generator is typically realized by counters, comparators and RS latches in the existing DPWM schemes [13,14]. In comparison, the proposed pulse width generation circuit is made up only of a counter and a comparator, where the width of the rising signal $\mathrm{R}$ is controlled by the MSBs of DCC (see Fig. 4(b)). Obviously, the complexity of the circuit is significantly reduced, and the stability can be enhanced simultaneously [20].

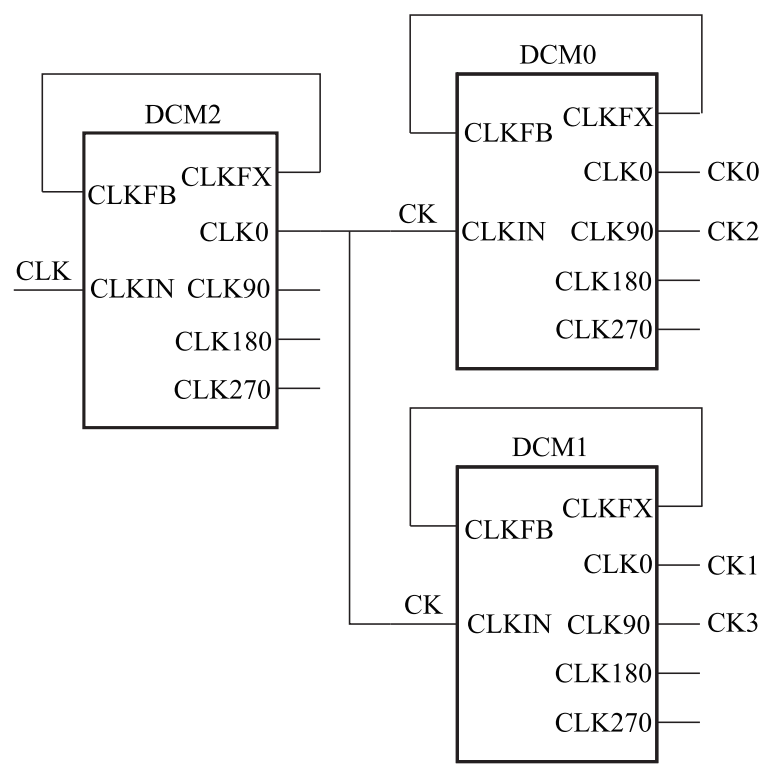

(a) Structure of the clock generation circuit

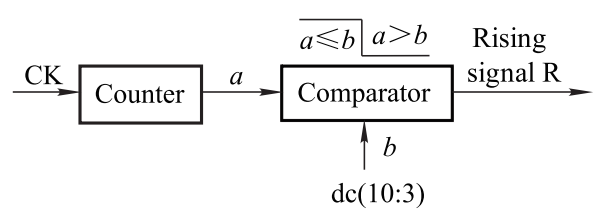

(b) Structure of the pulse width generation circuit

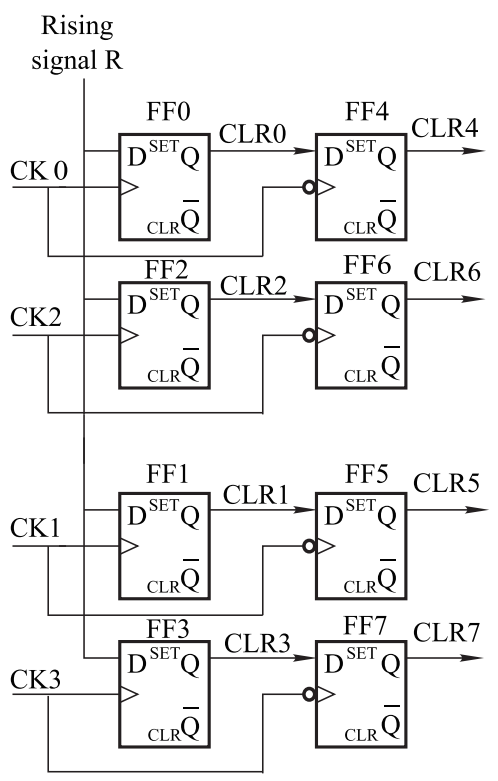

(c) Structure of the phase-shift delay circuit

Fig. 4 Key modules in DPWM based on embedded DCM

Phase-shift delay circuit in Fig. 1 is made up of eight D-triggers. As is shown in Fig. 4(c), triggers FF0, FF1, FF2 and FF3 work on the rising edge of CK0, CK1, CK2, CK3, while triggers FF4, FF5, FF6 and FF7 are driven by the falling edge of CK0, CK1, CK2, CK3. As a result, the output signals CLR0-CLR7 have a phase shift of $0^{\circ}, 45^{\circ}, 90^{\circ}, 135^{\circ}, 180^{\circ}, 225^{\circ}, 270^{\circ}$, and $315^{\circ}$ with respect to the rising signal $\mathrm{R}$, respectively.

\section{DPWM based on the digital programmable delay circuit}

The second method proposed in this paper uses the digital programmable delay circuit to construct controllable highresolution DPWM. As indicated in Fig. 5, the proposed DPWM architecture includes a clock generation circuit, a pulse width generation circuit, a digital programmable delay circuit, and a gate circuit. The main difference between the two proposed methods lies in that the phase-shift delay circuit in Fig. 1 is replaced by the digital programmable delay circuit in Fig. 5. More importantly, the delay accuracy of the DPWM is guaranteed by the well-designed placement and routing of LUTs in the digital programmable delay circuit. The detailed working principle of the DPWM will be described in the following.

The clock generation circuit operates on the input clock signal and exports the basic clock signal CK. As is demonstrated in Subsection 2.2, the pulse width generation circuit generates the rising signal $\mathrm{R}$ and sends it to the digital programmable delay circuit and the gate circuit. In particular, the digital programmable delay function is realized by a cascade of basic delay units (BDUs), where the BDU con- 
sists of a programmable data selector and a delay unit, in which the selector decides whether or not to perform the delay function by the control word.

After obtaining the delayed signal, the original and the delayed pulses are operated by the OR gate to generate the DPWM signal. Similarly as before, the rising edge of the pulse signal decides the rising edge of the DPWM signal, and the falling edge of the delayed signal determines the falling edge of the DPWM signal.

LUT queue provides a convenient way to implement the delay unit. The INIT attribute of the LUT defines the function of LUT. If the value of INIT is 32' h8000_0000, the output signal of the LUT is equal to the input signal. Due to the fact that FPGA devices usually use LUTs with four input terminals, each LUT is equivalent to an RAM with four address lines and an output terminal. For each LUT, the input terminals $\mathrm{A} 0, \mathrm{~A} 1, \mathrm{~A} 2$ are set to 0 , and the input terminal A3 is connected to the output of the front LUT in the queue (see Fig. 6(b)). This implies that the function of the LUT queue at this moment is not a logic operation but merely a fixed time delay. The rising signal $\mathrm{R}$ can now be delayed by the digital programmable delay circuit, generating the delay pulse width signals CLR0, CLR $1, \ldots$, CLR $2^{n}-1$. It is noteworthy that the placement and routing constraints play an important role in making the time delay controllable and accurate. Specifically, the layout and routing technology is employed to fix the selectors to the internal laterally adjacent LUT units in the FPGA. In addition to that, different delay units are fixed to the internal longitudinal adjacent LUT units in the FPGA. Theoretically speaking, the delay time for a single LUT is the resolution limit of the proposed DPWM and it varies with different devices. Nevertheless, considering the sensitivity of the asynchronous circuit to the power supply as well as to the ambient temperature, it is more advisable to design BDUs consisting of multiple LUTs. The gate circuit in the topright corner of Fig. 5 includes a selector and an OR gate, where the selector chooses one of the delayed pulse width signals as the falling signal $\mathrm{F}$ under the control of LSBs of DCC. The rising and the falling signals are combined by the OR gate circuit to generate the desired DPWM signal.

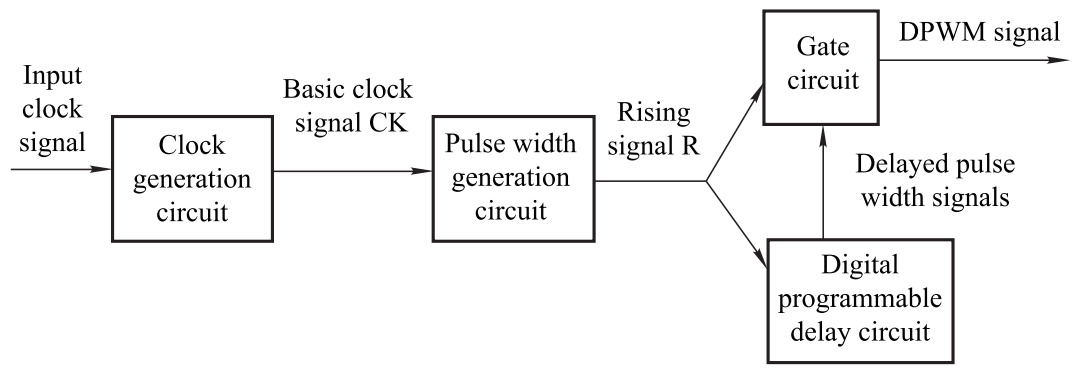

Fig. 5 DPWM based on digital programmable delay circuit

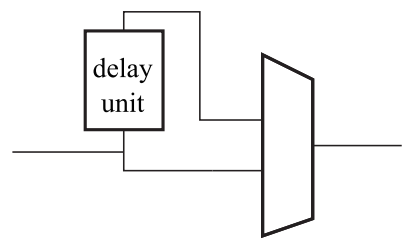

(a) Circuit diagram of BDU
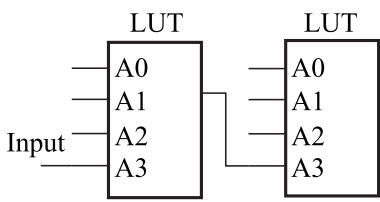

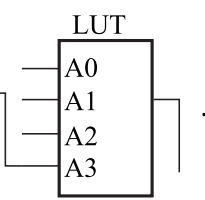

(b) LUT-based delay unit

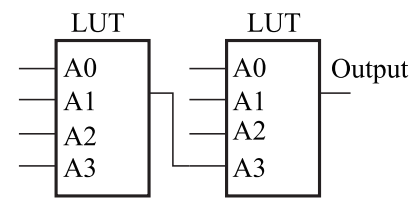

the LUT queue

\section{Experimental results}

This section further illustrates the implementations of DPWM based on the embedded DCM and digital programmable delay circuit. Fig. 7 presents the equipment used in the experiment.

Specifically, the proposed schemes are implemented on the Xilinx Artix-7 development board, where the basic clock signal CK operates at the frequency of $200 \mathrm{MHz}$. In order to obtain a quantitive observation of the waveform and the time step $\Delta t_{\mathrm{on}}$, the output of DPWM is connected to a high-performance oscilloscope ( $4 \mathrm{GHz}, 20 \mathrm{GSa} / \mathrm{s})$ produced by Rohde \& Schwarz. Firstly, three pulses with different widths are generated by the DCM-based DPWM, the corresponding DCCs are respectively " 00000110000 ", "00000110010" and "00000110100", where the low three bits are the LSBs. It is seen in Fig. 8 that the rising edges of the pulses are strictly aligned and the pulse width increment is 1250 ps between every two adjacent waveforms, meaning that the resolution of the DCM-based DPWM can reach up to $625 \mathrm{ps}$, which well coincides with the theoretical analysis. 


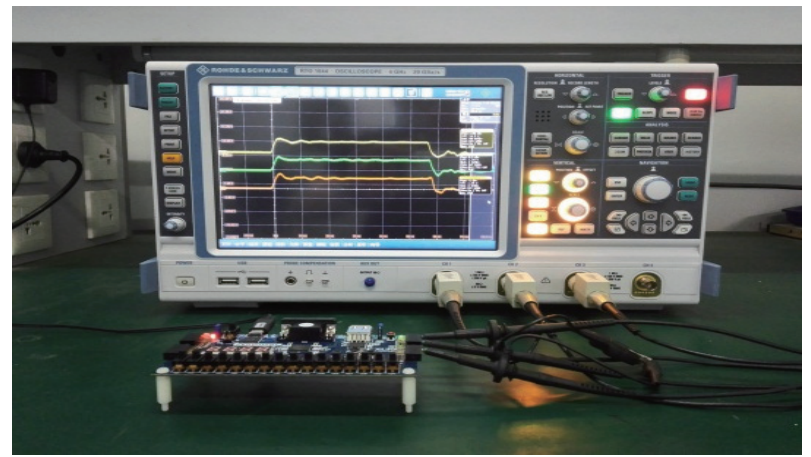

Fig. 7 The experimental equipment

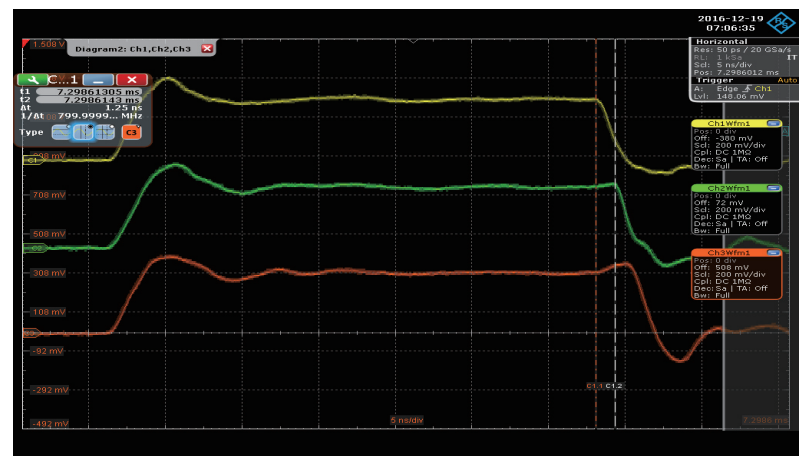

Fig. 8 Performance of the DCM-based scheme with different LSBs

The second example investigates the performance of DPWM based on the digital programmable delay circuit, where a single BDU is made up of seven LUTs. Fig. 9 shows the pulse width and the width increment of three pulses, where the DCCs are respectively set to "00101100000", "00101100010" and "00101-100100", in which the low three bits are the LSBs. It is clear that the rising edges of the pulses are strictly aligned and the pulse width increment is around $1 \mathrm{~ns}$ between every two adjacent waveforms. This in turn implies that the resolution of the DPWM can reach up to the level of about $500 \mathrm{ps,} \mathrm{which}$ coincides basically with the internal test result conducted by Xilinx.

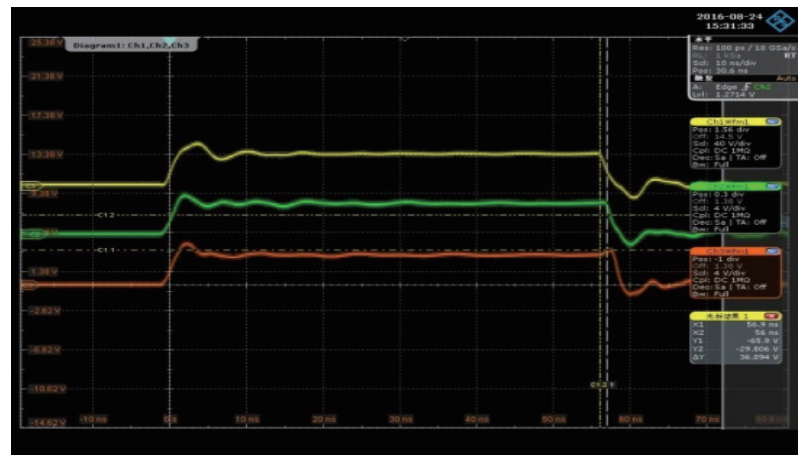

Fig. 9 Performance of the programmable delay circuit-based scheme with different LSBs
In the last example, pulse width increments of the DPWM based on the digital programmable delay circuit are calculated and plotted in Fig. 10, where the LSBs of the DCC signal have four bits. The horizontal axis represents 16 consecutive duty cycle commands and the vertical axis is the pulse width increment of the DPWM signal. A monotonic behavior can be observed on the oscilloscope and $\Delta t_{\text {on }}$ fluctuates around $500 \mathrm{ps}$, which is consistent with the previous experiment.

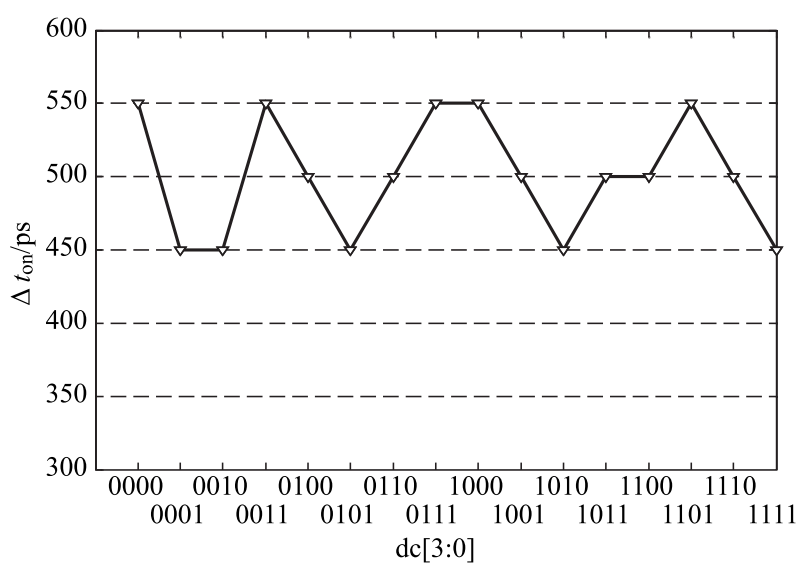

Fig. 10 Pulse width increment of the DPWM corresponding to different DCCs

\section{Conclusions}

Two DPWM implementations are proposed and verified in this paper. For the classical DCM-based approach, the structure is significantly simplified, and the stability is enhanced by incorporating an ordinary gate circuit. For lowcost applications, we propose to construct asynchronous DPWMs by using digital programmable delay circuits, where optimal placing and routing of LUT queues plays a vital role in realizing accurate and stable time delays.

\section{References}

[1] LUCÍA O, BARRAGAN L A, BURDÍO J M, et al. A versatile power electronics test-bench architecture applied to domestic induction heating. IEEE Trans. on Industrial Electronics, 2011, 58(3): $998-1007$.

[2] LUCÍA O, BURDÍO J M, BARRAGAN L A, et al. Seriesresonant multiinverter for multiple induction heaters. IEEE Trans. on Power Electronics, 2010, 25(11): 2860-2868.

[3] WANG F, SHEN W, BOROYEVICH D, et al. Voltage source inverter. IEEE Industry Applications Magazine, 2009, 15(2): $24-33$.

[4] SYED A, AHMED E, MAKSIMOVIC D, et al. Digital pulse width modulator architectures. Proc. of the 35th IEEE Annual Conference on Power Electronics, 2004: 4689-4695.

[5] PRODIC A, MAKSIMOVIC D, ERICKSON R W. Design and implementation of a digital PWM controller for a highfrequency switching DC-DC power converter. Proc. of the 27th IEEE Annual Conference on Industrial Electronics, 2001: $893-898$. 
[6] MAZUMDER S K, SARKAR T. Optically activated gate control for power electronics. IEEE Trans. on Power Electronics, 2011, 26(10): 2863 - 2886.

[7] SARNAGO H, MEDIANO A, LUCÍA O. High efficiency ACAC power electronic converter applied to domestic induction heating. IEEE Trans. on Power Electronics, 2012, 27(8): $3676-3684$.

[8] ORMAETXEA E, ANDREU J, KORTABARRIA I, et al. Matrix converter protection and computational capabilities based on a system on chip design with an FPGA. IEEE Trans. on Power Electronics, 2011, 26(1): 272-287.

[9] PONGIANNAN R K, PARAMASIVAM S, YADAIAH N. Dynamically reconfigurable PWM controller for three phase voltage source inverters. IEEE Trans. on Power Electronics, 2011, 26(6): $1790-1899$.

[10] JENG L F, CHIN H J, HUAN C P, et al. FPGA-based intelligent-complementary sliding-mode control for PMLSM servo-drive system. IEEE Trans. on Power Electronics, 2010, 25(10): $2573-2587$.

[11] LUCÍA O, URRIZA I, BARRAGAN L A, et al. Real-time FPGA-based hardware-in-the-loop simulation test bench applied to multiple output power converters. IEEE Trans. on Industry applications, 2011, 47(2): 853-860.

[12] COUGO B, MEYNARD T, GATEAU G. Parallel three-phase inverters: optimal PWM method for flux reduction in intercell transformers. IEEE Trans. on Power Electronics, 2011, 26(8): $2184-2191$.

[13] NAVARRO D, LUCÍA O, BARRAGAN L A, et al. Synchronous FPGA-based high-resolution implementations of digital pulse-width modulators. IEEE Trans. on Power Electronics, 2012, 27(5): 2515-2525.

[14] DE C A, TODOROVICH E. DPWM based on FPGA clock phase shifting with time resolution under $100 \mathrm{ps}$. Proc. of the IEEE Annual Power Electronics Specialists Conference, 2008: $3054-3059$.

[15] DE C A, TODOROVICH E. High resolution FPGA DPWM based on variable clock phase shifting. IEEE Trans. on Power Electronics, 2010, 25(5): $1115-1119$.

[16] BATARSEH M G, HOOR W A, HUANG L, et al. Windowmasked segmented digital clock manager-FPGA-based digital pulse-width modulator technique. IEEE Trans. on Power Electronics, 2009, 24(11): 2649-2660.

[17] SCHARRER M, HALTON M, SCANLAN T. FPGA-based digital pulse width modulator with optimized linearity. Proc. of the IEEE Applied Power Electronics Conference \& Exposition, 2009: 1220 - 1225.

[18] PETERCHEV A V, SANDERS S R. Quantization resolution and limit cycle in digitally controlled PWM converters. IEEE Trans. on Power Electronics, 2003, 18(1): 301-308.

[19] SABARINATH V, SIVANANDAM K. Design and implemen- tation of FPGA based high resolution digital pulse width modulator. Proc. of the IEEE International Conference on Communications and Signal Processing, 2013: 410-414.

[20] LI H T, ZHU X H, CHEN G. Design and application of Verilog HDL and FPGA. Beijing: National Defense Industry Press, 2013. (in Chinese)

\section{Biographies}

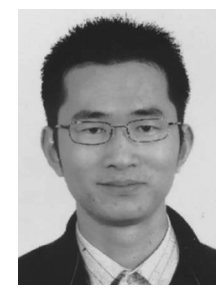

SONG Hu was born in 1980. He received his master degree in mechanical engineering from Nanjing University of Science and Technology in 2005. After that, he joined Nanjing Marine Radar Institute and worked as a system engineer. He is now a Ph.D. candidate in Nanjing University of Science and Technology. His research interests are radar system and high speed signal processing, with an emphasis on broadband array signal processing.

E-mail: songh_ee@163.com

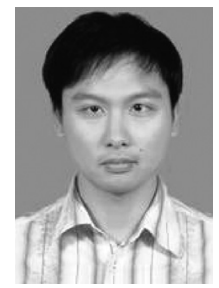

JIANG Naiti was born in 1984. He received his master degree in electronic engineering from Nanjing University of Information Engineering in 2010. He joined Nanjing Marine Radar Institute since 2010. He is now a Ph.D. student in Nanjing University of Information Science and Technology. His research interests are signal processing and data processing of marine radar system.

E-mail:Jnt724@126.com

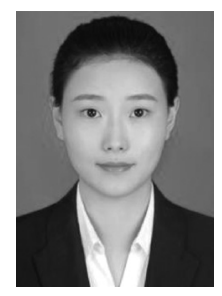

HU Shanshan was born in 1992. She received her master degree in electronic engineering from Nanjing University of Science and Technology in 2017. She joined Nanjing Metro company since 2017. She is a graduate student in Nanjing University of Science and Technology. Her research interest is radar signal processing.

E-mail: 787095030@qq.com

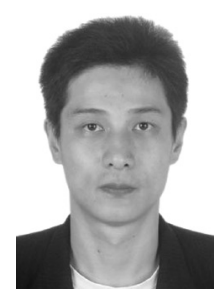

LI Hongtao was born in 1979. He received his $\mathrm{Ph} . \mathrm{D}$. degree in electronic engineering from Nanjing University of Science and Technology in 2012. He is now an associate professor in School of Electronic and Optical Engineering, Nanjing University of Science and Technology. His research interests are array signal processing and FPGA high speed design. E-mail: liht@njust.edu.cn 